\title{
In silico study of anthocyanin and ternatin flavonoids for the treatment of inflammation-related diseases using molecular docking analysis
}

\author{
${ }^{1}$ Wijaya, Y.T., ${ }^{1}$ Yulandi, A., ${ }^{1}$ Gunawan, A.W. and ${ }^{1,2 *}$ Yanti \\ ${ }^{1}$ Faculty of Biotechnology, Atma Jaya Catholic University of Indonesia, Jakarta 12930, Indonesia \\ ${ }^{2}$ Research Center for Indonesian Spices, Atma Jaya Catholic University of Indonesia, Jakarta 12930, \\ Indonesia
}

\begin{abstract}
Article history:
Received: 11 November 2019

Received in revised form: 24

November 2019

Accepted: 29 December 2019

Available Online: 15 January 2020
\end{abstract}

\section{Keywords:}

Anthocyanin flavonoid,

Ternatin flavonoid,

Inflammatory markers,

Anti-inflammatory activity,

Molecular docking assay

DOI:

https://doi.org/10.26656/fr.2017.4(3).378

\section{Introduction}

The cyclooxygenase (COX) activity is one of the major targets of non-steroidal anti-inflammatory drugs (NSAIDs), such as aspirin, ibuprofen, naproxen, and indomethacin. NSAIDs are common use drugs, both economically and clinically, but have the gastric side effect. COX-2 has more attention to the development of selective inhibitors, such as celecoxib and rofecoxib (Selinsky et al., 2001; Liu et al., 2019). Several reports on NSAIDs noted that about $20 \%$ of regular users of NSAIDs may cause duodenum or gastric ulcer (Wallace, 2001). Flavonoids consist of various classes such as anthocyanins, flavonols, flavones, flavanones, and isoflavones that have been reported to possess antiinflammatory activities. Butterfly pea (Clitoria ternatea) blue petal riched in ternatin anthocyanin has ability to suppress the nuclear factor kappa beta (NF-kB) translocation and inducible nitric oxide synthase (iNOS) expression in macrophages. In addition, $C$. ternatea ternatin anthocyanin can act as a drug or nutraceutical agent for protection against chronic inflammatory diseases by suppressing the excessive production of proinflammatory mediators secreted by macrophages, and as a promising candidate for cancer therapeutics (Nair et al., 2015; Srinivas et al., 2019). A study by Bukhari et al. (2015) demonstrated that flavonoids such as chalcone and pyrazoline exerted anti-inflammatory activity by attenuating the expression of $i N O S, C O X-2$, and other pro-inflammatory cytokines.

C. ternatea plant also exerts antioxidant property as a defense system to scavenge the reactive oxygen species (ROS) and protect the plant from destructive reactions (Sivaprabha et al., 2008). Antioxidant compounds in $C$. ternatea belong to anthocyanins that are known as phenolic antioxidants. Antioxidant and ROS have diverse roles in plant growth and in resistance to environmental stress (Zhang, 2019). ROS are continuously produced in plants as toxic byproducts of aerobic metabolism and rapidly detoxified. Some antioxidants are themselves free radical, donating 
electrons and neutralize the toxic free radicals.

Molecular docking is a computational chemistry method to study the rational drug design process. For example, this assay could be applied for studying ligandreceptor interaction mode and exploring several chemical data through in silico studies. Docking result using the software could be used to predict about binding energy of enantiomers (Ramírez and Caballero, 2016; Lamie and Azmey, 2019). In silico and in vivo studies of $C$. ternatea anthocyanin as an anti-inflammatory candidate has not been explored yet, while comparison data from both studies will be needed for determining novel antiinflammatory drug with fewer side effects, effective, and efficient. In this research, in silico study of anthocyanin and ternatin flavonoids as the potential binding ligands to the active site of inflammatory markers, including COX1, COX-2, iNOS, and MPO using molecular docking analysis compared to that of ibuprofen as a native ligand for COX-1 was evaluated

\section{Materials and methods}

In silico study was conducted at DNA Technology and Bioinformatics Laboratory, Faculty of Biotechnology, Atma Jaya Catholic University of Indonesia, Jakarta (Indonesia). The automatic docking study was carried out using Protein-Ligand ANT System (PLANTS, version 4.6, Universität Konstanz, Germany) as implemented through the graphical user interface YASARA. Protein crystal structures of COX-1, COX-2, iNOS, and MPO complex (PDB codes: 1EQG, 3Q7D, $4 \mathrm{UX6}$, and 5FIW; Figure 1) were retrieved from the RSCB Protein Data Bank (http://www.rcsb.org/pdb/ home/home.do). The PDB archives contained the drugs bound to the receptors. Ligand molecule structures of ibuprofen, anthocyanin flavonoid, and ternatin flavonoid (Figure 2) were obtained from MolView(http:// molview.org/). All bound water and ligands were eliminated from the protein and the polar hydrogen using YASARA (http://www.yasara.org/).

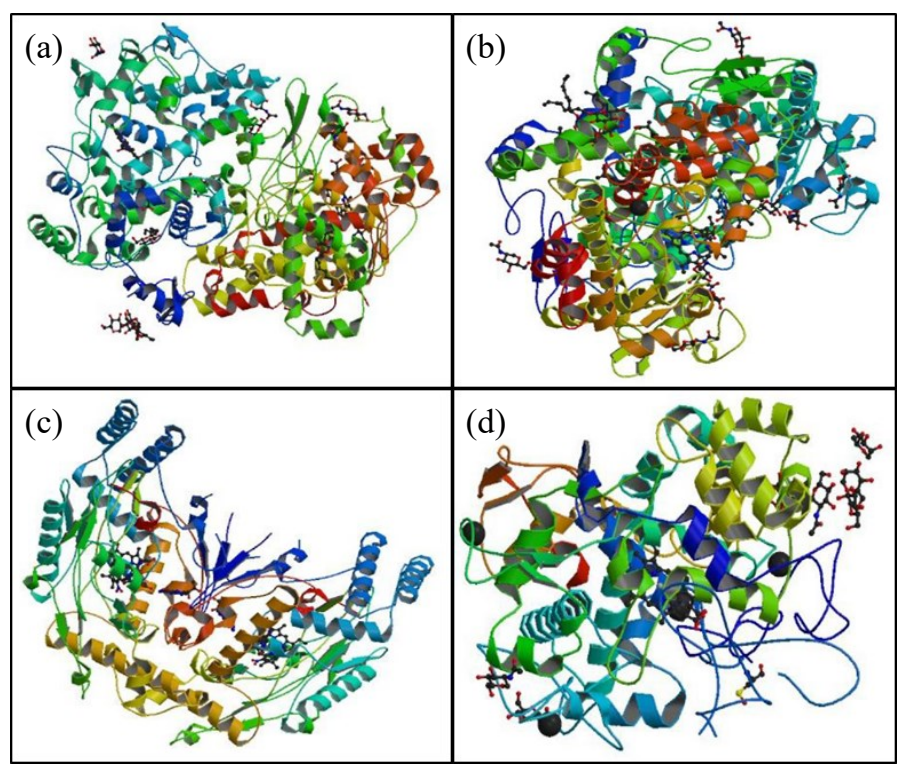

Figure 1. Protein crystal structures of COX-1(1EQG.pdb; a), COX-2 (3Q7D.pdb; b), iNOS (4UX6.pdb; c), and MPO (5FIW.pdb; d) as inflammatory markers for studying ligandprotein binding using molecular docking analysis. COX, cyclooxygenase; iNOS, inducible nitric oxide synthase; MPO, myeloperoxidase

The other binding energy scoring was predicted by different algorithms using AutoDock Vina (version 1.1.2, The Scripps Research Institute, USA). All commands were put in one configuration file for parameter receptor and ligand. This configuration file was used as the guidance for running AutoDock Vina. For its input and output, AutoDock Vina used the PDBQT molecular structure file format. PDBQT files were generated (interactively or in batch mode) and viewed by using MGLTools (http:// mgltools.scripps.edu/).

\section{Results and discussion}

In silico data (Figures 3-5) showed the docking results performed with PLANTS for all ligand compounds (ibuprofen, anthocyanin flavonoid, and ternatin flavonoid). The values of protein binding energy for all ligand compounds were listed in Table 1. Docking analysis was performed by calculating binding energy (a)

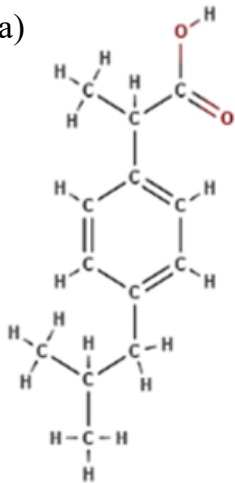

(b)<smiles>C=CC=Cc1ccc2ccccc2[o+]1</smiles>

(c)

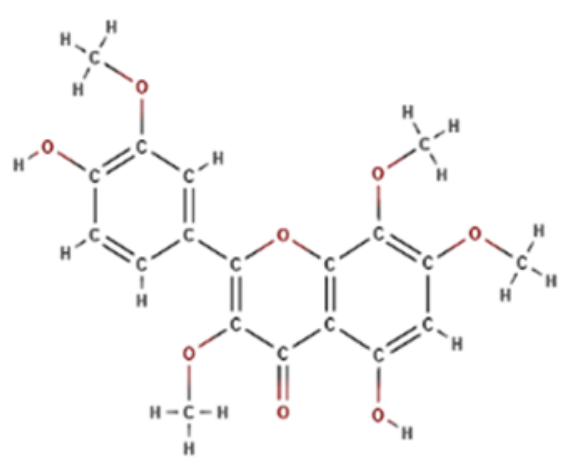

Figure 2. Ligand molecules including ibuprofen (a), anthocyanin (b), and ternatin (c). as inhibitors for studying ligand-protein binding using molecular docking analysis. COX, cyclooxygenase; iNOS, inducible nitric oxide synthase; MPO, myeloperoxidase. 


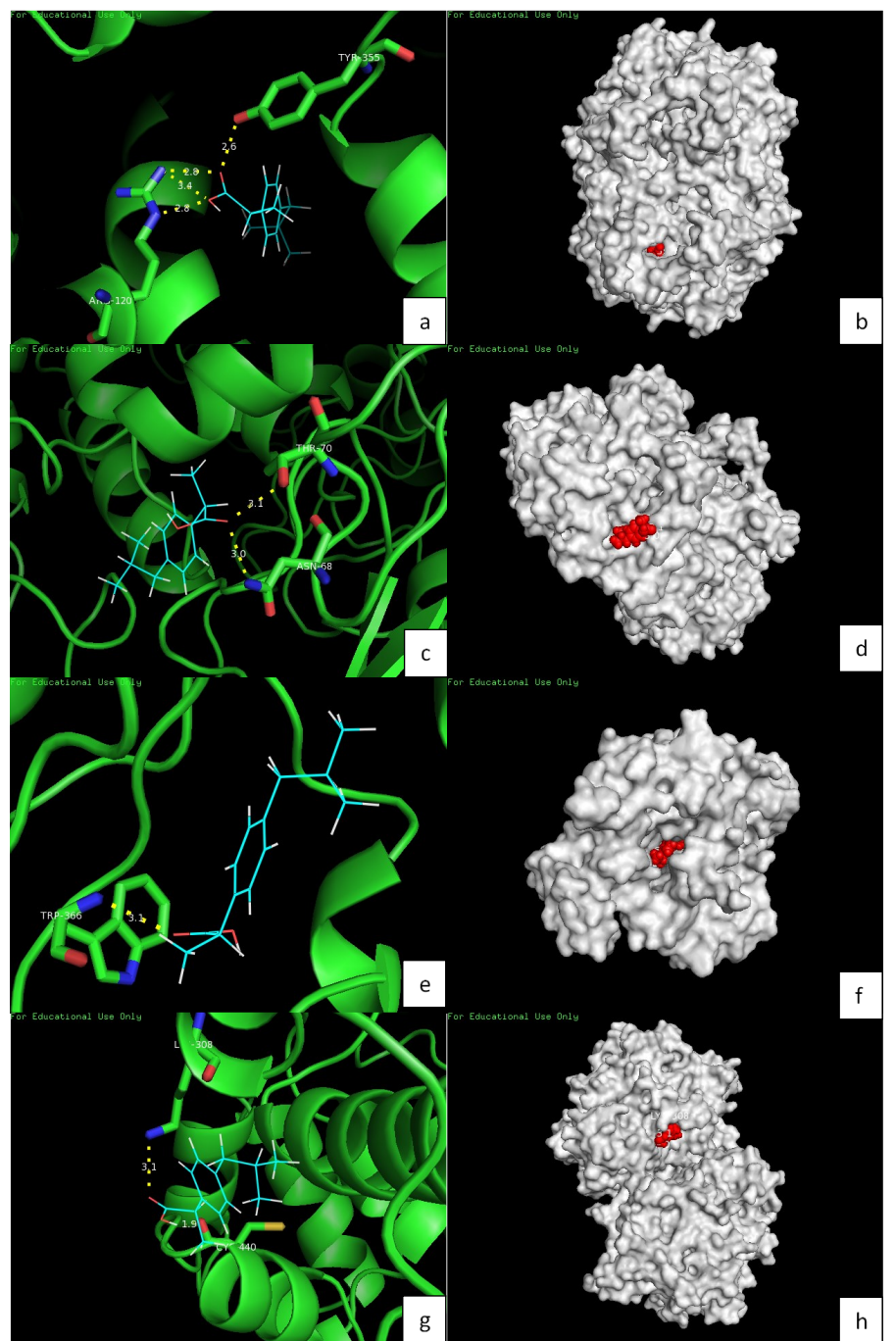

Figure 3. Bonding complex between ibuprofen with COX-1 (1EQG.pdb; a and b), COX-2 (3Q7D.pdb; $c$ and d), iNOS (4UX6.pdb; e and f), and MPO (5FIW.pdb; g and h) using AutoDock Vina. COX, cyclooxygenase; iNOS, inducible nitric oxide synthase; MPO, myeloperoxidase.

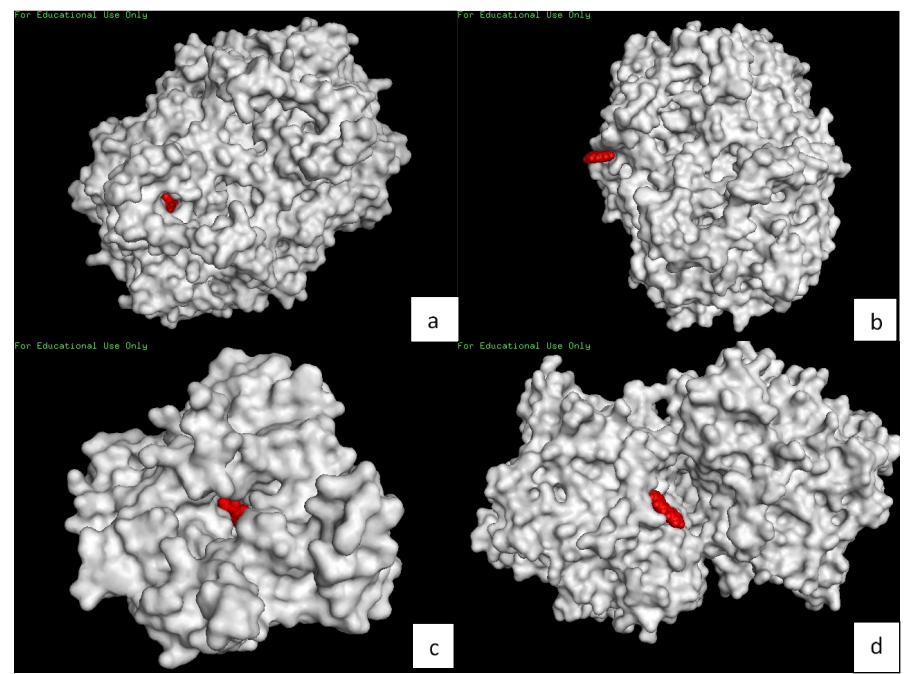

Figure 4. Bonding complex between anthocyanin with COX-1 (1EQG.pdb; a), COX-2 (3Q7D.pdb; b), iNOS (4UX6.pdb; c), and MPO (5FIW.pdb; d) using AutoDock Vina. COX, cyclooxygenase; iNOS, inducible nitric oxide synthase; MPO, myeloperoxidase.

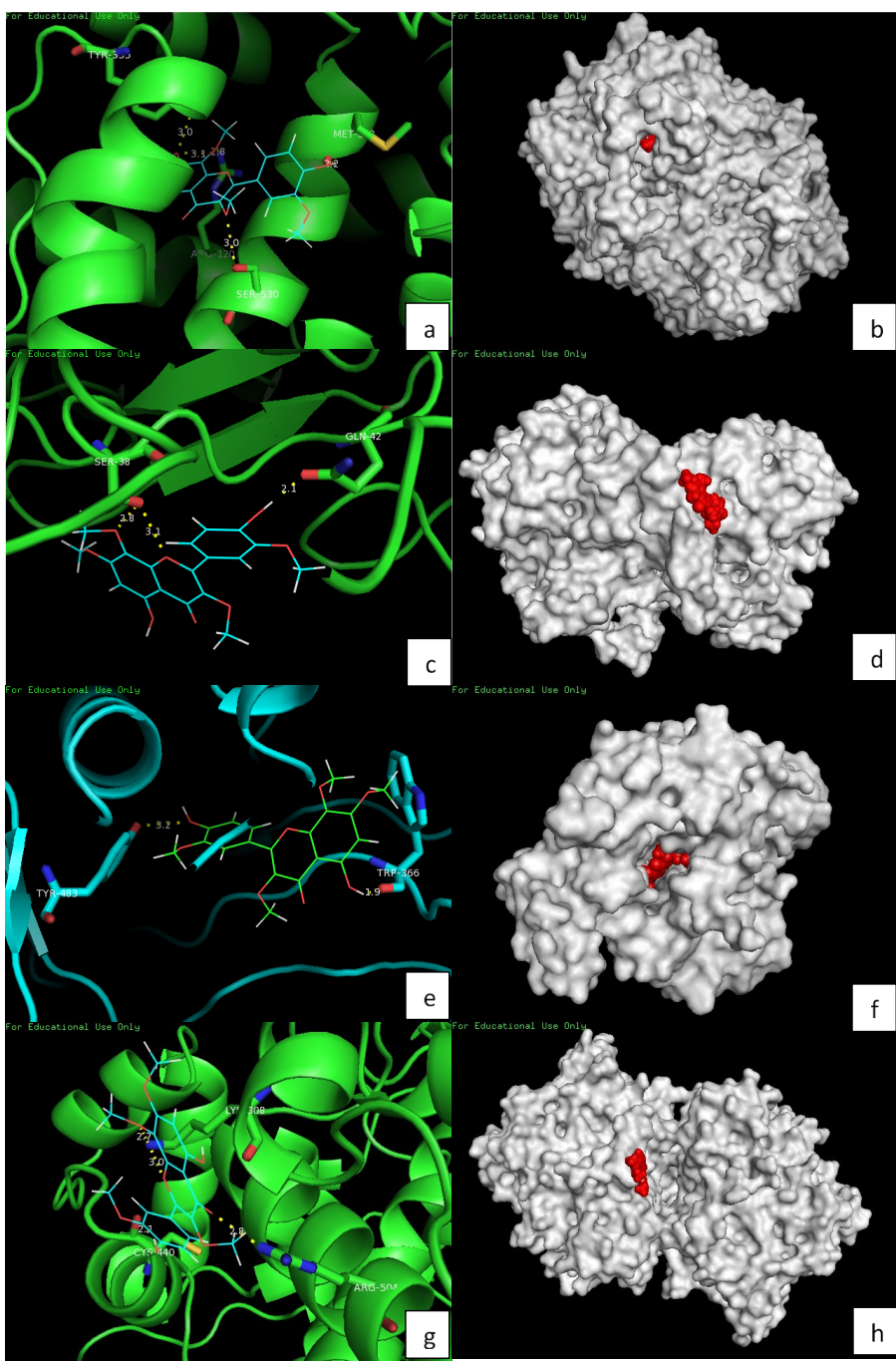

Figure 5. Bonding complex between ternatin with COX-1 (1EQG.pdb; a and b), COX-2 (3Q7D.pdb; c and d),iNOS (4UX6.pdb; e and f), and MPO (5FIW.pdb; g and h) using AutoDock Vina. COX, cyclooxygenase; iNOS, inducible nitric oxide synthase; MPO, myeloperoxidase.

Table 1. Protein-ligand binding energy with PLANTS

\begin{tabular}{lcccc}
\hline \multirow{2}{*}{ Compound } & \multicolumn{4}{c}{ Binding energy (kcal/mol) } \\
\cline { 2 - 5 } & COX-1 & COX-2 & iNOS & MPO \\
\hline Ibuprofen & -80.60 & -50.71 & -81.95 & -54.35 \\
Anthocyanin & -78.47 & -55.62 & -93.05 & -52.56 \\
Ternatin & -56.16 & -57.23 & -95.54 & -52.42 \\
\hline
\end{tabular}

COX, cyclooxygenase; iNOS, inducible nitric oxide synthase; MPO, myeloperoxidase

Table 2. Protein-ligand binding energy with AutoDock Vina

\begin{tabular}{lcccc}
\hline \multirow{2}{*}{ Compound } & \multicolumn{4}{c}{ Binding energy (kcal/mol) } \\
\cline { 2 - 5 } & COX-1 & COX-2 & iNOS & MPO \\
\hline Ibuprofen & -7.90 & -6.80 & -7.50 & -6.00 \\
Anthocyanin & -9.10 & -7.90 & -8.30 & -6.90 \\
Ternatin & -7.50 & -7.70 & -7.70 & -7.00 \\
\hline
\end{tabular}

COX, cyclooxygenase; iNOS, inducible nitric oxide synthase; MPO, myeloperoxidase 
values among ligand compounds and proteins regulating inflammatory responses (COX-1, COX-2, MPO, and iNOS). PLANTS was used to visualize docking among ibuprofen with COX-1 and COX-2. Ibuprofen was found to be a native ligand for COX-1. Our results also indicated that ibuprofen effectively bound to the active site of COX-1 with minimum binding energy $\left(\Delta G_{\mathrm{b}}=\right.$ $80.60 \mathrm{kcal} / \mathrm{mol})$ but did not bind to the active site of $\operatorname{COX}-2\left(\Delta G_{\mathrm{b}}=-50.71 \mathrm{kcal} / \mathrm{mol}\right)$.

Table 1 shows that ibuprofen was a potent inhibitor for COX-1, as the binding energy indicated its binding potential to the active site of COX-1. Ternatin was a potential inhibitor for $\mathrm{COX}-2$, in which the binding site for this protein is long, slender with a hydrophobic channel extending from the membrane-binding region. A second cavity branched off from the main channel led to the cyclogenase active site was observed in COX-2, which bound greatly to ternatin. Anthocyanin and ternatin were bound into the active site of COX-1.

Based on PLANTS analysis (Table 1), anthocyanin had similar minimum binding energy $\left(\Delta G_{\mathrm{b}}=-78.47\right.$ $\mathrm{kcal} / \mathrm{mol}$ ) with that of ibuprofen. Among the ligand compounds, lower COX-2 binding energy for ternatin $\left(\Delta G_{\mathrm{b}}=-57.23 \mathrm{kcal} / \mathrm{mol}\right.$ with PLANTS) compared to those of anthocyanin and ibuprofen $\left(\Delta G_{\mathrm{b}}=-55.62 \mathrm{kcal} /\right.$ $\left.\mathrm{mol}, \Delta G_{\mathrm{b}}=-50.71 \mathrm{kcal} / \mathrm{mol}\right)$. These data showed COX-1 bound effectively to small compounds, while COX-2 bound to larger compounds due to the size of its active site.

All ligand compounds had the ability to bind the active site of both COX-1 and COX-2 (Figures 3-5). The 2D-images of binding mode between each compound and the target proteins were demonstrated. The active pocket of COX-1 interacted with ibuprofen via Tyr-355 and Arg-120. COX-1 interaction with ternatin was mediated by Tyr-355, Arg-120, Ser-530, and Met-522. COX-2 showed interaction with ibuprofen via Thr-70 and Asn-68. COX-2 interaction with ternatin was shown at Ser-38 and Gln-42. The binding site of iNOS interacted with ibuprofen through Trp-366, and with ternatin at Trp-366 and Tyr-483. The MPO showed interaction with ibuprofen through Lys-308 and Cys-440, and interaction with ternatin via Lys-308, Cys-440, and Arg-504. The most substantial differences between these three inhibitors were found in the substituents on the phenyl ring which lie at the end of the molecule from the carboxylic acid group.

Docking studies based on AutoDock Vina showed that the three ligand compounds showed various binding energies (Table 2). Anthocyanin was able to inhibit COX -1 , COX-2, and iNOS; therefore, it showed the highest potential for anti-inflammatory efficacy. However, anthocyanin binding to MPO was less effective. Anthocyanin and ternatin could effectively bind to the active site of COX-1. Based on PLANTS and AutoDock Vina analysis, values for binding energy and optimum binding energy for the active site of COX-1 were variable.

PLANTS results demonstrated that ibuprofen was the best compound for binding to the active site of COX1, while AutoDock Vina results showed that anthocyanin was the best. This might be due to the fact that both software used different algorithms to calculate binding energy at protein active sites. All tested ligands (ibuprofen, anthocyanin, and ternatin) bound COX-2 active site, which was located at the apex of a long narrow hydrophobic channel extending from the membrane-binding surface to the center of protein (Figures 3-5). It should be noted that ibuprofen contains an isobutyl substituent on its phenyl ring, rather than a second aromatic group, and furthermore, this compound is not halogenated.

In the present study, in silico modeling based on ligand-protein binding energy was done. The in-silico data generated through PLANTS and AutoDock Vina demonstrated that ibuprofen, anthocyanin, and ternatin bound to COX-1 and COX-2 active sites (Figures 3-5 and Tables 1-2). These three compounds shared structural similarity, in which one or more phenyl rings are present in all of them. Compound size affects minimum binding energy for binding to the active site of the target protein (Tables 1 and 2). Docking poses generated by the PLANTS and AutoDock Vina can be directly loaded into PyMOL. Moreover, docking poses resulted in meta-information, i.e. the docking score for structure analysis of score relationships. All docking poses were ranked according to their docking scores, in which lower score indicated lower binding energy. Structure-based docking methods rank molecules via a scoring function that is calculated based on their inner methods (Yang et al., 2019). In addition, the previous study also showed that AutoDock Vina was a more efficient option for stimulating protein docking with macrolides and analogues of intermediate size ligand compared to other docking programs, such as Glide 6.6, AutoDock 4.2 and DOCK 6.5 (Castro-Alvarez et al., 2017).

Both PLANTS and AutoDock Vina showed consistent molecular docking results for ibuprofen and COX-1, as well as for its competitors (anthocyanin and ternatin flavonoids) and its rapidly reversible mechanism for COX inhibition. Mechanism of inhibition by ibuprofen was observed for COX-1 and COX-2, involving ligand binding to both proteins near the solvent 
-accessible opening of the hydrophobic channel, followed by a fast translocation of the inhibitor along the length of the channel, all of which associated with the COX active site. These results showed that ibuprofen binding orientation to COX-1 and COX-2 was similar. The carboxyl group of ibuprofen formed the hydrogen bond with Arg-120 and Tyr-355 at the COX-1 active site. Our results are in line with the previous study by Viegas et al. (2011), who conducted crystallography analysis of COX-1 and ibuprofen binding analysis using saturation transfer difference NMR (STD-NMR).

To date, there has yet been model for ibuprofen binding to COX-2 or anthocyanin and ternatin flavonoids binding to COX-1, COX-2, iNOS, and MPO. Interestingly, based on our in silico data generated through PLANTS (Figures 3-5), we were able to propose binding orientation and energy for ibuprofen binding to COX-1. This is also supported by the consistency of phenyl ring in ibuprofen for the docking arrangement and similarities among the positioning of aromatic ring in anthocyanin and ternatin with that of the phenyl ring in ibuprofen.

\section{Conclusion}

In silico analysis indicated that molecular docking of ternatin flavonoid bound effectively to the active site of most inflammatory proteins, including COX-1, COX-2, and iNOS, and MPO. Based on its binding energy, anthocyanin flavonoid was able to inhibit COX-1, COX2 , and iNOS. These data suggest that anthocyanin and ternatin flavonoids may act as potential inflammatory inhibitors as well as ibuprofen for treatment of inflammatory-related diseases.

\section{Conflict of Interest}

The authors declare no conflict interest.

\section{Acknowledgement}

This research was funded by the Decentralization Grant from General Directorate of Higher Education, Ministry of Research, Technology and Higher Education, Republic of Indonesia.

\section{References}

Castro-Alvarez, A., Costa, A.M. and Vilarrasa, J. (2017). The performance of several docking programs at reproducing protein-macrolide-like structures. Molecules, 22(1), $136 . \quad$ https:// doi.org/10.3390/molecules22010136

Lamie, P.F. and Azmey, A.F. (2019). Synthesis and biological evaluation of tetrazole derivatives as TNF $-\alpha$, IL-6 and COX-2 inhibitors with antimicrobial activity: Computational analysis, molecular modeling study and region-specific cyclization using 2D NMR tools. Bioorganic Chemistry, 92, 103301. https://doi.org/10.1016/j.bioorg.2019.103301

Liu, D., Wang, Y., Li, L., Zhao, H., Li, L., Liu, Y., Jiang, H., Li, X. and Zhang, R. (2019). Celecoxib protects hyperoxia-induced lung injury via NF- $\kappa \mathrm{B}$ and AQP1. Frontiers in Pediatrics, 7(228), 1-11. https:// doi.org/10.3389/fped.2019.00228

Nair, V., Bang, W.Y., Schreckinger, E., Andarwulan, N. and Zevallos, L.C. (2015). Protective role of ternatin anthocyanins and quercetin glycosides from butterfly pea (Clitoria ternatea leguminosae) blue flower petals against lipopolysaccharide (LPS)-induced inflammation in macrophage cells. Journal of Agricultural and Food Chemistry, 63(28), 63556365. https://doi.org/10.1021/acs.jafc.5b00928

Ramírez, D. and Caballero, J. (2016). Is it reliable to use common molecular docking methods for comparing the binding affinities of enantiomer pairs for their protein target? International Journal of Molecular Sciences, 17(4), 525. https://doi.org/10.3390/ ijms 17040525

Selinsky, B.S., Gupta, K., Sharkey, C.T. and Loll, P.J. (2001). Structural analysis of NSAID binding by prostaglandin $\mathrm{H} 2$ synthase: time-dependent and time -independent inhibitors elicit identical enzyme conformations. Biochemistry, 40(17), 5172-5180. https://doi.org/10.1021/bi010045s

Sivaprabha, J., Supriya, J., Sumathi, S., Padma, P.R., Nirmaladevi, R. and Radha, P. (2008). A study on the levels of nonenzymic antioxidants in the leaves and flowers of Clitoria ternatea. Ancient Science of Life, 27(4), 28-32.

Srinivas, B.K., Shivamadhu, M.C., Siddappaji, K.K., Krishnappa, D.K. and Jayarama, S. (2019). Angiosuppressive effects of bio-fabricated silver nanoparticles synthesis using Clitoria ternatea flower: an in vitro and in vivo approach. Journal of Biological Inorganic Chemistry, 24(7), 1115-1126. https://doi.org/10.1007/s00775-019-01721-x

Viegas, A., Manso, J., Corvo, M.C., Marques, M.M. and Cabrita, E.J. (2011). Binding of ibuprofen, ketorolac, and diclofenac to COX-1 and COX-2 studied by saturation transfer difference NMR. Journal of Medicinal Chemistry, 54(24), 8555-8562. https:// doi.org/10.1021/jm201090k

Wallace, J.L. (2001). Pathogenesis of NSAID-induced gastroduodenal mucosal injury. Best Practice and Research, Clinical Gastroenterology, 15(5), 691703. https://doi.org/10.1053/bega.2001.0229 
Yang, M., Tao, B., Chen, C., Jia, W., Sun S., Zhang, T. and Wang, X. (2019). Machine learning models based on molecular fingerprints and an extreme gradient boosting method lead to the discovery of JAK2 inhibitors. Journal of Chemical Information and Modeling, 59(12), 5002-5012. https:// doi.org/10.1021/acs.jcim.9b00798

Zhang, X., Wei, X., Wang, M., Zhu, X., Zhao, Y., Wei, F. and Xia, Z. (2019). Overexpression of NtabDOG1L promotes plant growth and enhances drought tolerance in Nicotiana tabacum. Plant Science, 287, 110186. https://doi.org/10.1016/ j.plantsci.2019.110186 